\section{Unilateral inferior oblique muscle myectomy and recession in the treatment of inferior oblique muscle overaction: a longitudinal study \\ T Shipman and J Burke}

\begin{abstract}
Background The comparable long-term outcomes of inferior oblique muscle myectomy and recession for the treatment of superior oblique underaction (in primary position and straight right and left gaze) have not been well documented in the literature.

was $15 \Delta$ or more. An improvement occurred in both groups immediately after surgery and in many throughout the follow-up period represented by a continuing drift towards orthotropia, but there was a recurrence of the hyperdeviation in some of the recession patients.
\end{abstract} The purpose of this study was to compare longitudinally these two procedures in a similar, patient population with binocular single vision, when both operations were performed by the same surgeon, with a minimum follow-up period of 12 months. Methods A total of 24 patients who randomly underwent either a unilateral myectomy (at the temporal border of the inferior rectus muscle) or a standard recession for inferior oblique muscle overaction associated with long-standing superior oblique underaction were evaluated preoperatively at 2 weeks, 4 months, and 12 months postoperatively by the same orthoptist.

Results A total of 23 patients met the study criteria, (12 myectomies and 11 recessions). All but one patient had demonstrable binocular single vision. The average preoperative hyperdeviation in contralateral gaze was $\mathbf{2 6 . 5}$ prism dioptres $(\Delta)$ in the myectomies and $20 \Delta$ in the recessions. This was reduced at $\mathbf{1 2}$ months postoperatively to $1.75 \Delta$ in the myectomies and to $3 \Delta$ in the recessions. Both procedures were largely self-grading, so that the larger the preoperative hyperdeviation, the greater the effect of surgery.

Conclusions Single inferior oblique muscleweakening procedures were effective in the vast majority of patients, even when the preoperative primary position hyperdeviation
Eye (2003) 17, 1013-1018. doi:10.1038/

sj.eye. 6700488

Keywords: inferior oblique recession; inferior oblique myectomy; superior oblique underaction; inferior oblique overaction

\section{Introduction}

Inferior oblique muscle overaction may cause a socially noticeable vertical hypertropia of the affected eye in primary position and contralateral gaze and/or symptomatic diplopia and asthenopia. Surgical management is often required to either improve alignment and/or relieve symptoms. The most commonly performed inferior oblique muscle-weakening procedures are inferior oblique myectomy and inferior oblique recession. The surgical decision appears to be primarily based on individual experience and preference.

It is widely reported that both procedures result in a self-grading operation, so that the greater the preoperative hyperdeviation, the larger the correction obtained postoperatively. ${ }^{1-4}$ It has also been documented that inferior oblique muscle-weakening procedures in isolation may be less effective if there is significant preoperative hyperdeviation in primary position and in ipsilateral gaze.,
Department of

Ophthalmology

Royal Hallamshire Hospital

Sheffield, UK

Correspondence:

J Burke

Department of

Ophthalmology

Royal Hallamshire Hospital

Glossop Road

Sheffield S10 2JF, UK

Tel: + 441142712036

Fax: + 441142713682

E-mail: julie.d.taylor@

sth.nhs.uk

Received: 19 July 2002; accepted in revised form: 17 December 2002 
There are a number of pre-existing retrospective studies that were designed to provide comparative data on inferior oblique muscle-weakening operations. All these studies include patients with and without binocular single vision. Costenbader and Kertesz ${ }^{6}$ retrospectively compared myectomies, recessions, and disinsertions on 131 eyes of patients with diverse strabismus aetiologies and binocularities undergoing isolated inferior oblique muscle surgery, and in 359 patients with combined inferior oblique and horizontal muscle surgery. They qualitatively graded the inferior oblique muscle overaction pre- and postoperatively according to the severity of the overaction of the adducting eye into three groups, as slight, moderate, and marked. No consideration was given to the primary position measurements. They concluded that the postoperative outcome was generally comparable for all procedures.

Cooper and Sandall ${ }^{7}$ retrospectively compared 54 recessions and 102 disinsertion procedures on the inferior oblique muscle performed on 107 patients with and without binocularity; 63 of whom had simultaneous horizontal muscle surgery; they also adjudged the effect of both procedures to be comparable.

Parks ${ }^{8}$ reviewed the earlier literature and prospectively evaluated 319 patients ranging in age from 6 months to 17 years with a diverse range of binocularity with symmetrical bilateral inferior oblique muscle overaction. A total of 224 had combined horizontal muscle surgery and 66 others had horizontal muscle surgery either prior to or following the inferior oblique muscle surgery, with a minimum of 2 years follow-up. For each patient, each eye had one of the four different weakening procedures. Two criteria were used to evaluate the effects of surgery (creation of a primary position vertical deviation and the change in the eye's elevating power in adduction). Parks found inferior oblique muscle recession to be the most effective procedure. He observed a persistent inferior oblique muscle overaction in $37 \%$ of patients and inferior oblique muscle underaction in $8 \%$ of patients after an inferior oblique muscle myectomy, and $13 \%$ incidence of inferior oblique muscle adhesive syndrome when the myectomy was performed at the inferior oblique muscle insertion. Parks ${ }^{9}$ subsequently describes how certain approaches to the inferior oblique muscle may predispose to an adhesive syndrome rather than the myectomy itself.

The aim of our study was to examine longitudinally and compare the amount of change produced by a single inferior oblique muscle myectomy or recession to the measured hyperdeviation in primary position, right and left gaze, and in the amount of inferior oblique muscle overaction at 2 weeks, 4 months, and 12 months postoperatively in a more aetiologically and binocularly similar group of patients than hitherto reported. We sought to determine if either procedure gave a better long-term outcome in the measured hyperdeviation and inferior oblique muscle function in the three horizontal cardinal gaze positions in patients with binocular single vision.

\section{Methods}

All patients presenting to the department with either symptom producing and/or a socially noticeable, unilateral overacting inferior oblique muscle were included in the study. No patient had previously undergone any muscle surgery and no other surgery was performed at the same time as the inferior oblique muscle-weakening procedure. No patient had any surgical or prismatic intervention during the follow-up period. Patients were excluded from the study if they did not cooperative with all tests, had visual acuity of 20/60 or worse in either eye, or failed to attend any of the postoperative assessment visits.

A full orthoptic assessment was performed on each patient by the same orthoptist and specifically included visual acuity testing, cover test, alternate prism cover test using loose prisms at $6 \mathrm{~m}$ in primary position, and right and left gaze. Ocular movements were recorded using diagrammatic representation and numerical evaluation. ${ }^{10}$ Assessment of binocular function, suppression, Hess charts, and field of binocular single vision were also recorded when appropriate.

All patients were randomly selected to have either a single inferior oblique muscle myectomy at the temporal border of the inferior rectus muscle or a standard $10 \mathrm{~mm}$ inferior oblique muscle recession performed by the same surgeon (JB).

An inferotemporal conjunctival fornix incision was made; the conjunctiva and tenons were opened separately in layers. The inferior oblique muscle was identified and hooked under direct vision. The inferior oblique was cleared of its surrounding intermuscular septa from its insertion to near the temporal border of the inferior rectus muscle. The inferior oblique muscle was clamped adjacent to its insertion and disinserted from the globe between the artery clamp and its insertion. For an inferior oblique muscle recession, a double-armed 6-0 vicryl suture was then passed through the muscle adjacent to the artery clamp with lock-bites at either pole. The two ends of the 6-0 vicryl suture were then passed through scleral tunnels $2-3 \mathrm{~mm}$ apart with the anterior suture inserted $3 \mathrm{~mm}$ posteriorly and $2.5 \mathrm{~mm}$ lateral to the temporal pole of the inferior rectus muscle. For a myectomy, a second artery clamp was used to clamp the muscle near the temporal border of the inferior rectus muscle. The muscle was transected adjacent and 
temporal to the second clamp. Haemostasis was achieved prior to removing the clamp. The inferior oblique muscle was then observed and its retraction facilitated into tenons capsule overlying the inferior rectus muscle so that its stump was no longer in direct contact with the sclera. For both procedures, the conjunctiva and tenons were then closed in separate layers using interrupted 8-0 vicryl sutures.

\section{Results}

All patients had a decompensated long-standing unilateral superior oblique underaction. Of 24 consecutive patients in the study, 23 were successfully followed up for at least 1 year, 12 myectomies and 11 recessions (Table 1 ). The 12 th recession patient who was asymptomatic in the early postoperative period failed to

Table 1 Preoperative results in patients undergoing inferior oblique muscle surgery

\begin{tabular}{|c|c|c|c|c|}
\hline \multirow[t]{2}{*}{ Patient } & \multicolumn{2}{|c|}{ Preoperative } & \multirow[t]{2}{*}{$B V$ status } & \multirow[t]{2}{*}{ Age (years) } \\
\hline & IO function & SO function & & \\
\hline $1 \mathrm{M}$ & +3 & -1.5 & 1 & 22 \\
\hline $2 \mathrm{M}$ & +2 & -2.5 & 1 & 27.9 \\
\hline $3 \mathrm{M}$ & +3 & -2.5 & 1 & 30 \\
\hline $4 \mathrm{M}$ & +2 & -2 & 1 & 13 \\
\hline $5 \mathrm{M}$ & +3.5 & -2.5 & 1 & 147 \\
\hline $6 \mathrm{M}$ & +2 & -2 & 1 & 31.4 \\
\hline $7 \mathrm{M}$ & +4 & -3.5 & 2 & 45.8 \\
\hline $8 \mathrm{M}$ & +2.5 & -3 & 1 & 65.5 \\
\hline $9 \mathrm{M}$ & +3 & -2.5 & 1 & 12 \\
\hline $10 \mathrm{M}$ & +3 & -1.5 & 1 & 15.5 \\
\hline $11 \mathrm{M}$ & +3 & -3 & 1 & 14.5 \\
\hline $12 \mathrm{M}$ & +3 & -2 & 3 & 77 \\
\hline $13 R$ & +3 & -2 & 1 & 13.7 \\
\hline $14 \mathrm{R}$ & +3 & -2 & 1 & 32 \\
\hline $15 R$ & +3 & -2 & 1 & 13.7 \\
\hline $16 \mathrm{R}$ & +2 & -2 & 2 & 69 \\
\hline $17 R$ & +3 & -2.5 & 1 & 23.4 \\
\hline $18 \mathrm{R}$ & +3 & -2.5 & 1 & 33.5 \\
\hline $19 R$ & +3 & -2 & 1 & 21.5 \\
\hline $20 R$ & +2.5 & -2 & 1 & 23 \\
\hline $21 R$ & +3 & -2 & 1 & 24.1 \\
\hline $22 \mathrm{R}$ & +3 & -1 & 1 & 36.5 \\
\hline $23 R$ & +3 & -2 & 1 & 23 \\
\hline
\end{tabular}

$\mathrm{IO}$, inferior oblique muscle; $\mathrm{SO}$, superior oblique muscle; $\mathrm{BV}$, binocular vision; 1, bifoveal BV; 2 , monofoveal BV with microtropia; 3, suppression with constant manifest deviation; $\mathrm{M}$, myectomy; $\mathrm{R}$, recession. attend for long-term follow-up. All patients had overacting inferior oblique muscles of 2 units or more; the corresponding versional superior oblique underactions ranged from -1.5 to -3.5 units (Table 1 ). On ductions, the superior oblique underaction was minimal to normal. Out of 12 patients in the myectomy group, 11 demonstrated binocular vision. Of these, 10 patients had bifoveal binocular single vision (acuity of 6/ 6 or better in each eye, no manifest strabismus, fusional amplitudes demonstrable, stereoacuity of $60^{\prime \prime}$ or better), one had a microstrabismus (stereoacuity of 85 " of arc and a small angle manifest strabismus of less than $10 \Delta$ present on cover testing), and the remaining patient had suppression. All patients in the recession group demonstrated binocular vision, 10 with bifoveal binocular single vision, one with microstrabismus (stereoacuity of $85^{\prime \prime}$ of arc). There were no surgical complications. All patients were satisfied with the surgical result at the 12 months postoperative assessment.

\section{Postoperative reduction of hyperdeviation}

Preoperative contralateral gaze measurements ranged from 12 to $35 \Delta$ in the myectomy group with an average (median) of $26.5 \Delta$ (Table 2). The measurements were similar in the recession group, with a range of 13-30 $\Delta$ preoperatively, the average (median) was $20 \Delta$. No statistically significant difference was evident between the two groups preoperatively (contralateral gaze measurement, $P=0.177$; preoperative superior oblique underaction, $P=0.88$, Mann-Whitney test). At the final 12-month postoperative assessment, the median contralateral hyperdeviation was $1.75 \Delta$ in the myectomy group and $3 \Delta$ in the recession group (Table 2 ).

Figures 1 (myectomies) and 2 (recessions) show the median amount of hyperdeviation preoperatively and at 2 weeks, 4 months, and 12 months postoperatively in ipsilateral gaze, primary position and contralateral gaze in both groups and show the gradual return towards orthotropia in all positions of gaze during the 12 months follow-up.

When the two groups are compared with each other at 12 months postoperatively at $6 \mathrm{~m}$, a recession on average (median) resulted in $8 \Delta$ reduction of vertical deviation in

Table 2 Median angles of hyperdeviation $(\Delta)$ in contralateral gaze at $6 \mathrm{~m}$

\begin{tabular}{lcccc}
\hline & Preop & 2 weeks postop & 4 months postop & 12 months postop \\
\hline $\begin{array}{l}\text { Myectomy } \\
\text { (range })\end{array}$ & +26.5 & +10.5 & +7.5 & +1.75 \\
$\begin{array}{l}\text { Recession } \\
\text { (range })\end{array}$ & $(+12$ to +35$)$ & $(+4$ to +18$)$ & +0 to +16$)$ & $(-5$ to +16$)$ \\
\hline
\end{tabular}



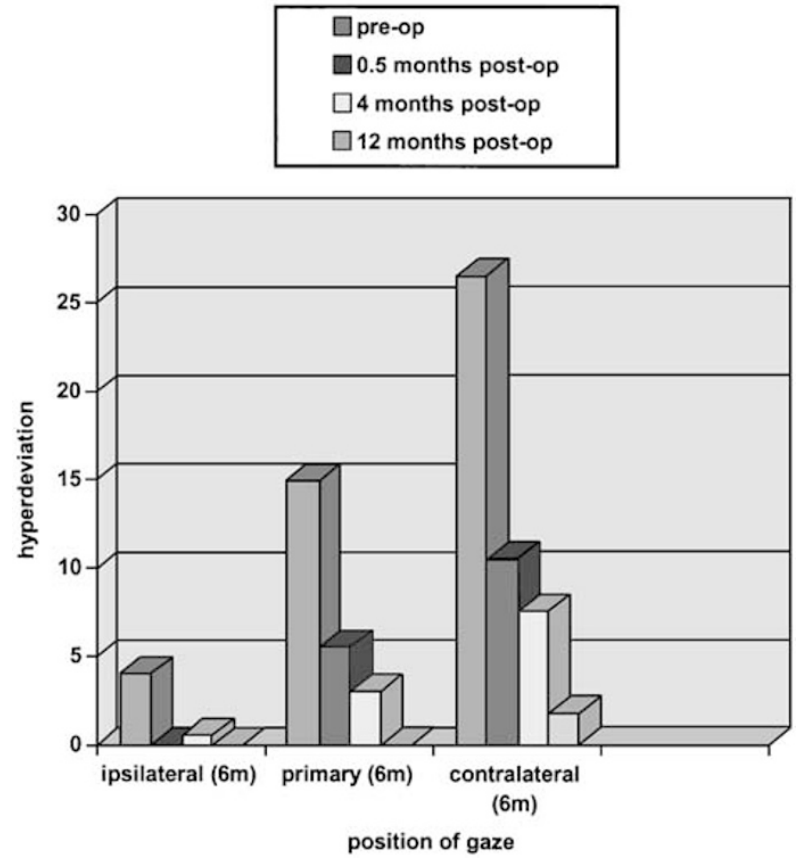

Figure 1 Median hyperdeviation $(\Delta)$ in the myectomy group preoperatively and at $0.5,4$, and 12 months postoperatively.

primary position, a myectomy in $14 \Delta$, while in contralateral gaze, a recession resulted in $16 \Delta$ of reduction and a myectomy in $18.5 \Delta$. In ipsilateral gaze, there was an average reduction of $4.5 \Delta$ in the myectomy group and $5 \Delta$ in the recessions.

\section{Change in hyperdeviation from 2 weeks to 12 months postoperatively}

The reductions in the hyperdeviation that occurred during the postoperative period, from 2 weeks to 12 months, are shown in Table 3. Statistical significance was achieved in both groups (contralateral gaze measurements: myectomies: $P<0.001$; recessions $P=0.048$, Wilcoxon's rank test). The myectomy group showed the greatest amount of change represented as a drift towards orthotropia and this was statistically significant $(P=0.015)$. In the recession group, three patients demonstrated a recurrence in their hyperdeviation from 4 to 12 months postoperatively, whereas the majority of the myectomies (nine patients) continued to improve during this time while the remaining three myectomies remained stable.

\section{Postoperative change in oblique muscle function}

Preoperative inferior oblique muscle function values ranged from +2 to +3 units of overaction in the recession group with a median of +3 units and from +2

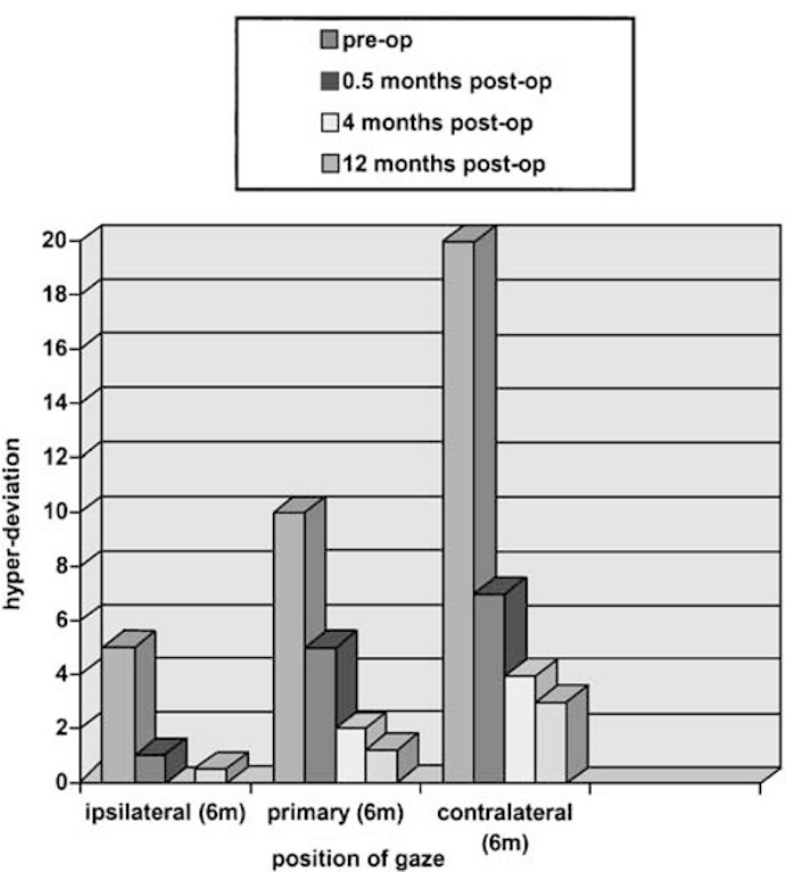

Figure 2 Median hyperdeviation $(\Delta)$ in the recession group preoperatively and at $0.5,4$, and 12 months postoperatively.

Table 3 Average change of hyperdeviation $(\Delta)$ in each group 2 weeks to 12 months postoperatively

\begin{tabular}{lccc}
\hline & $\begin{array}{c}\text { Ipsilateral } \\
\text { gaze }\end{array}$ & $\begin{array}{c}\text { Primary } \\
\text { position }\end{array}$ & $\begin{array}{c}\text { Contralateral } \\
\text { gaze }\end{array}$ \\
\hline $\begin{array}{l}\text { Myectomy } \\
\text { (range })\end{array}$ & 0 & +5 & +7 \\
Recession & $(-4$ to +8$)$ & $(+3$ to +10$)$ & $(1$ to +13$)$ \\
(range $)$ & 0 & +3 & +2 \\
\hline
\end{tabular}

+ Values denote a reduction of the hyperdeviation $(\Delta)$; -Values denote a recurrence of the hyperdeviation $(\Delta)$.

to +4 units overaction in the myectomy group with a median of +3 units also. There was no statistical difference preoperatively between either group, $(P=0.831$ Mann-Whitney test).

At 12 months postoperatively, there was an average (median) reduction in inferior oblique muscle overaction of 2 units in both groups. The overall change in inferior oblique muscle function from 2 weeks to 12 months was an average of 0.5 unit decrease in both the groups.

Similarly, there was a significant change in superior oblique muscle function following surgery. In the myectomies, the median preoperative versional underaction was -2.5 units, while at 12 months the postoperative underaction had reduced to -0.25 units. In the recessions, the preoperative versional underaction was -2 units, while the postoperative underaction was 0 at 12 months. The median amount of improvement in 
muscle function preoperatively to 12 months postoperatively was 1.75 units in the myectomies and 1.5 units in the recessions.

The median amount of improvement during the postoperative period was 1 unit in the myectomies and 0 unit in the recessions. This further improvement in superior oblique muscle function in the myectomies during this time was significant (myectomies: $P<0.001$; recessions: $P=0.059$ ).

There were six patients with preoperative primary position hypertropias of $15 \Delta$ or more (median $20 \Delta$, range $16-25 \Delta$ ). At 12 months postoperatively, the median postoperative primary position deviation was $1 \Delta$ (range 0-6 $\Delta$ ). There were four with preoperative ipsilateral hypertropias of $10 \Delta$ or more. At 12 months postoperatively the ipsilateral deviation ranged from 0 to $4 \Delta$ (range 10-15 $\Delta$ ). It was evident that single-muscle surgery, whether myectomy or recession, is effective in significantly decreasing the hypertropia in all gaze positions, even in those patients with a significant amount of primary or ipsilateral gaze hypertropia.

\section{Discussion}

In this study, both surgical procedures were effective, so that good primary position alignment was achieved and symptoms were eliminated in binocular cases. All patients and parents of affected teenagers were satisfied with the results of surgery throughout the 12 months follow-up. It was evident that uniform surgery tended to produce a variable self-titrating amount of correction of the hyperdeviation in both groups in all three gaze positions, even in the larger preoperative deviations, so that the larger the preoperative hyperdeviation, the greater the amount of correction obtained postoperatively, but the myectomy resulted in the greatest reduction of the hyperdeviation and the most consistently favourable outcome.

The average reduction of the hyperdeviation in the myectomy group at 12 months postoperatively in primary position was $14 \Delta$. Toosi and Von Noorden ${ }^{11}$ found a mean reduction of $11.9 \Delta$ of hyperdeviation in primary position and relatively little difference between the alignment in primary position and in the field of action of the inferior oblique or superior oblique muscles, whereas we found an increased amount of correction in contralateral gaze of $18.5 \Delta$, and this correction was dynamic and continued throughout the 12 month followup period. Helveston and Haldi ${ }^{12}$ similarly described a greater reduction of $20 \Delta$ of hyperdeviation in the field of action of the inferior oblique muscle from weakening a single inferior oblique muscle, which compares more favourably with our results (but they did not report statistical data).
In our study, a recession reduced the hyperdeviation by a median of $8 \Delta$ in primary position and by $16 \Delta$ in contralateral gaze. Cooper and Sandall ${ }^{7}$ stated that a measured recession will decrease the hyperdeviation by $6.88 \Delta$ in primary position and by $12.3 \Delta$ in the field of action of the overacting inferior oblique muscle. This compares well with Kutschke and Scott ${ }^{3}$ who found a reduction of $6.9 \Delta$ in primary position and $15.6 \Delta$ in contralateral gaze. Mittleman and Folk ${ }^{13}$ reported a decrease of $9 \Delta$ from a 10-12 $\mathrm{mm}$ measured recession.

If the hyperdeviation at 12 months postoperatively was examined in our study, the average deviation in contralateral gaze was $1.75 \Delta$ in the myectomies and $3 \Delta$ in the recession, both results appearing excellent. However, this difference between the amount of correction obtained from a myectomy compared with a recession in our study was significant, in contralateral gaze $P=0.047$ and in primary position $P=0.042$. This small but additional improvement in measured hyperdeviation provided a better result in terms of improved cosmesis and function.

The small amount of change that occurred after the immediate postoperative period, from 2 weeks to 12 months, was statistically significant, but only in the myectomy group where a further $7 \Delta$ of reduction of the hyperdeviation occurred $(P<0.001)$, there was no overall change in the recession group $(P=0.06)$. This difference between the two groups was also statistically significant $(P=0.013)$. The inferior oblique muscle function values also mirrored this trend so that most of the myectomies demonstrated a reduced inferior oblique overaction with improved superior oblique function between 2 weeks and 12 months postoperatively, whereas during the same period, the recessions followed a more variable course. It is possible that this happens because of the different anatomical and physiological effects of myectomy and recession of the inferior oblique muscle. In the myectomy technique, we employ one that permanently alters the length of the muscle and the torque on the globe as the eye attempts to move further into the field of action of the inferior oblique. During the postoperative period of muscle sequelae, this seemingly results in a progressive effect on alignment that eventually stabilises. In a recession the muscle is moved nearer to its origin and equatorially, producing slack in the muscle. The effect may be more likely to decrease with time and probably more so in its field of action as the muscle undergoes a period of gradual but variable length-tension adaptation and alignment outcome.

In this longitudinal study of teenagers and adults with binocular single vision, a myectomy does appear to confer a more satisfactory, long-term outcome as most patients demonstrated small but further improvements in their ocular alignment following the immediate 
postoperative period. Some of the recession patients also continued to improve with time but this was less predictable. Del Monte and Parks ${ }^{14}$ found when treating marked inferior oblique overaction (ie grade $4+$ ) of varying aetiologies that inferior oblique muscle denervation and extirpation appeared to be a more effective operation than a $14 \mathrm{~mm}$, inferior oblique recession for patients with $4+$ inferior oblique overaction over a mean follow-up period of 20.8 months. Gonzalez $^{15}$ has extended the use of the denervation/ myectomy procedure to moderate (ie +2 and +3 ) inferior oblique overaction with good effect. However, Gonzalez observed at least a $20 \%$ permanent overcorrection rate with this procedure in cases with mild (ie +1 ) inferior oblique overaction.

All patients preoperatively demonstrated an incomitant hypertropia, the deviation being significantly larger in contralateral gaze as would normally be expected, yet the vast majority of patients were rendered concomitant and successfully treated in all positions of gaze postoperatively, even when the ipsilateral preoperative hyperdeviation was greater than $15 \Delta$. It has been reported that the presence of a significant primary position preoperative hypertropia is more likely to be associated with undercorrection postoperatively when single-muscle procedures are employed..$^{3,5}$ In this study, both procedures reduced an overacting inferior oblique muscle, whether mild or marked to a normal state in most cases. There was only one patient (case number 12, Table 1A) who had a residual deviation of $4 \Delta$ in ipsilateral gaze ( $6 \Delta$ in primary position, $13 \Delta$ in contralateral gaze). This was the only patient who did not demonstrate any binocular single vision, which may well be a significant factor.

The numbers of patients included in each surgical group were small and this may have affected the power of the study; although, each group had a similar number of patients and the average preoperative hyperdeviation and overacting inferior oblique muscle values were alike for both myectomies and recession groups. All patients were examined by the same orthoptist and surgery was performed in the previously described method by the same surgeon, providing a homogenous study population.

This longitudinal study in a specific group of patients with preoperative binocular single vision and superior oblique muscle underaction compares the efficacy of these two procedures in the three positions of gaze over a 12-month follow-up period. From the early and mediumterm results, it appears that both procedures are effective in significantly reducing the hyperdeviation and overacting inferior oblique muscle. They additionally highlight that the inferior oblique muscle myectomy may well be the procedure of choice giving a better and more predictable longer term outcome at least in this specific but common subgroup of patients with superior oblique underaction and measurable binocularity.

\section{References}

1 Harcourt B, Almond S, Freedman H. The efficacy of inferior oblique myectomy operations. In: Mein J, Moore S (eds). Orthoptics, Research and Practice. Transactions of the Fourth International Orthoptic Congress, 1979, Berne. Kimpton: London, 1981, pp. 20-23.

2 Davis G, McNeer KW, Spencer RF. Myectomy of the inferior muscle. Arch Ophthalmol 1986; 104: 855-858.

3 Kutschke PJ, Scott WE. The effect of inferior oblique muscle recession in the treatment of unilateral superior oblique palsy. Am Orthoptic J 1994; 44: 98-102.

4 Morad Y, Weinstock VM, Kraft SP. Outcome of inferior oblique recession with or without vertical rectus recession for unilateral superior oblique paresis. Binocular Vision 2001; 16: 23-27.

5 Reynolds JD, Biglan AW, Hiles DA. Congenital superior oblique palsy in infants. Arch Ophthalmol 1984; 102: 1503-1505.

6 Costenbader FD, Kertesz E. Relaxing procedures of the inferior oblique - a comparative study. Am J Ophthalmol 1964; 57: 276-280.

7 Cooper EL, Sandall GS. Recession versus free myotomy at the insertion of the inferior oblique muscle. J Pediatr Ophthalmol 1969; 6: 6-10.

8 Parks MM. The weakening surgical procedures for eliminating overaction of the inferior oblique muscle. Am J Ophthalmol 1972; 73: 107-122.

9 Parks MM. Causes of the Adhesive Syndrome. Symposium on strabismus. Transactions of the New Orleans Academy of Ophthalmology. The C.V. Mosby Company: St. Louis, 1978, pp 269-279.

10 Doughty DD, Lenarson LW, Scott WE. A graphic portrayal of versions. Perspect Ophthalmol 1978; 2: 55-59.

11 Toosi SH, Von Noorden GK. Effect of isolated inferior oblique muscle myectomy in the management of superior oblique muscle palsy. Am J Ophthalmol 1979; 88: 602-608.

12 Helveston EM, Haldi BA. Surgical weakening of the inferior oblique. Int Ophthalmol Clin 1976; 16: 113.

13 Mittleman D, Folk ER. The evaluation and treatment of superior oblique muscle palsy. Trans Am Acad Ophthalmol Otolaryngol 1976; 81: 893-898.

14 Del Monte M, Parks MM. Denervation and extirpation of the inferior oblique an improved weakening procedure for marked overaction. Ophthalmology 1983; 90: 1178-1183.

15 Gonzalez C. Discussion of denervation and extirpation of the inferior oblique. Ophthalmology 1983; 90: 1184-1185. 